Publications of the Astronomical Society of the Pacific

95:289-292, May 1983

\title{
OBSERVATIONS OF THE LINE PROFILE OF PASCHEN ALPHA IN 3C 273
}

\author{
K. SELLGREN, B. T. SOIFER, G. NEUGEBAUER, AND K. MATTHEWS \\ Palomar Observatory, California Institute of Technology, Pasadena, California 91125 \\ Received 1983 January 6
}

\begin{abstract}
Observations of the line profile of hydrogen Paschen $\alpha$ have been made in the quasar 3C 273. The line profile is the same, within the uncertainties, as the line profiles of $L \alpha, \mathrm{H} \beta$, and C IV observed in 3C 273.

Key words: quasars-3C 273-infrared spectra-line profiles
\end{abstract}

\section{Introduction}

The explanation of the anomalous hydrogen-line ratios in quasars has sparked considerable theoretical and observational effort over the last few years. The heart of the problem is the $L \alpha / \mathrm{H} \beta$ line ratio, which is observed to be about a factor of 10 less in quasars than the predictions of case $B$ recombination theory, while other line ratios, such as the $\mathrm{H} \alpha / \mathrm{H} \beta$ ratio, appear more nearly normal. An accompanying puzzle is the observation that the profiles of lines arising in widely disparate ionization conditions are similar. In the theoretical picture currently invoked to explain the observations of quasar lines, and recently reviewed by Davidson and Netzer (1979), the broad emission lines originate in numerous small clouds which contain within them a range of ionization conditions, so that each cloud emits all the lines seen in the broad-line-emitting regions of quasars, with the observed line ratios being to first order the same in each cloud. The linewidths in each cloud are characteristic of the thermal velocity of the gas within the cloud, with a temperature of $1-2 \times 10^{4} \mathrm{~K}$, while the observed broad linewidths of the quasar are due to mass motions of the many clouds within the broad-line-emitting region.

Current theoretical models (Kwan and Krolik 1979, 1981; Canfield and Puetter 1980) predict that different lines will be emitted by the clouds with varying degrees of anisotropy. For example, $L \alpha$ should emerge only through the cloud face directed toward the ionizing source, while other lines should be radiated more isotropically. These theoretical models would therefore predict that observations of the profiles of various lines might distinguish between models of the kinematics of the broad-line-emitting clouds. To date, no real difference has been observed between profiles of different lines, including $L \alpha$ (Davidson and Netzer 1979).

Previous investigations of the line profiles of quasars, as reviewed by Davidson and Netzer (1979), have been made in the visual and ultraviolet portions of the spectrum, where many of the lines of interest are predicted by current models to have large optical depths in the quasar clouds. We have extended line profile observations to infrared wavelengths, and have measured with $750 \mathrm{~km} \mathrm{~s}^{-1}$ resolution the line profile of hydrogen Paschen $\alpha$ (rest wavelength $1.875 \mu \mathrm{m}$ ) in the archetypal quasar, 3C $273(z=0.158)$. This affords an opportunity to test whether the similarity of line profiles of very optically thick lines such as $L \alpha$ and $H \beta$ applies also to less optically thick lines such as $P \alpha$. Additionally, the low relative extinction in the infrared, compared to that in the visual and ultraviolet, makes it possible to use the observed $P \alpha$ line profile to determine if the profiles of lines at shorter wavelengths have been substantially altered by extinction within the broad-line-emitting region.

\section{Observations}

The observations of 3C 273 were made on 1980 January 3 and 1980 January 4 on the 200-inch (5-m) Hale telescope of Palomar Observatory, using a single-stage piezoelectrically scanned Fabry-Perot interferometer coupled to an InSb detector. Low-resolution filtering was provided by a cooled, circular variable filter wheel with 5\% resolution. The spectrometer and data-acquisition system were similar to those described by Nadeau (1980), except that the Fabry-Perot plates were placed directly in the converging $f / 72$ beam of the Gregorian focus. The spectrum was scanned continuously over slightly more than 1 order every $10 \mathrm{~s}-20 \mathrm{~s}$, with calibration spectra of a krypton lamp obtained typically every 32 scans. The alignment of the Fabry-Perot plates was typically checked every 30 minutes by visual inspection of the $5461 \AA$ line of a mercury lamp. The total integration time was $1600 \mathrm{~s}$ on the first night and $4800 \mathrm{~s}$ on the second night. One order corresponded to 15,400 $\mathrm{km} \mathrm{s}^{-1}$ on the first night, and to $16,200 \mathrm{~km} \mathrm{~s}^{-1}$ on the second night. The instrumental resolution on these two nights was respectively 710 and $800 \mathrm{~km} \mathrm{~s}^{-1}$.

The observations were made with a diaphragm $5^{\prime \prime}$ in diameter. Sky subtraction was provided by a wobbling secondary mirror. Three stars were observed to calibrate the instrumental transmission, BS $3982(\mathrm{~B} 7 \mathrm{~V})$ and BS 4983 (G0 V) on the first night, and BS 4689 (A2 V) on 
the second night. These stars should be featureless in the velocity range observed, except for Brackett $\gamma$ absorption at a velocity of $-830 \mathrm{~km} \mathrm{~s}^{-1}$ with respect to the predicted central velocity of Paschen $\alpha$ in 3C 273. The results of Elias (1978) predict that $B \gamma$ should be approximately of equal strength in BS 3982 and BS 4689, and considerably weaker in BS 4983.

The instrumental transmission was found to vary strongly across the velocity range observed, due to the nonuniform transmission of the circular variable filter wheel. Furthermore the instrumental transmission as a function of velocity was different on the two nights, due to the difference in resolution. This required that the spectra, after being corrected for drifts in the velocity calibration and coadded, be corrected separately for the instrumental transmission on each night. The instrumental transmission on the first night was derived from the observations of BS 4983, which was assumed to have negligible $B \gamma$ absorption. On the second night the instrumental transmission was derived from BS 4689, with correction made for its $B \gamma$ absorption by a comparison of the instrumental transmissions derived for BS 3982 and BS 4983 on the previous night. The $2.2 \mu \mathrm{m}$ magnitudes of BS 4983 and BS 4689 were taken from Elias et al. (1982). The final spectrum of 3C 273 was produced by dividing the spectrum of each night by the instrumental transmission for that night, and then adding the spectra together weighted by the integration times.

\section{Results}

The spectrum of Paschen $\alpha$ in 3C 273 is shown in Figure 1, with each data point representing a bin $20 \mathrm{~km} \mathrm{~s}^{-1}$ wide. The instrumental resolution is $\sim 750 \mathrm{~km} \mathrm{~s}^{-1}$, which can be seen to be a small fraction of the observed

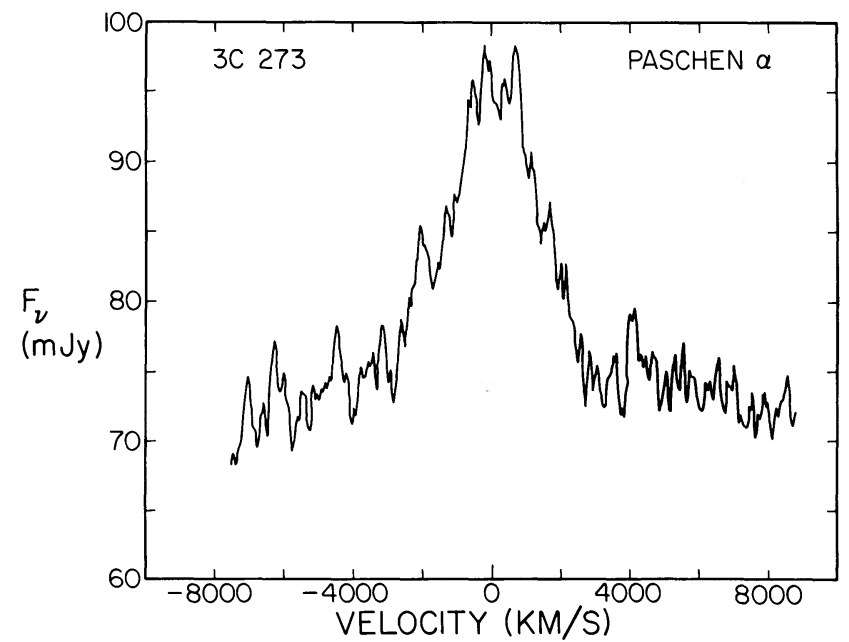

Fig. 1-The line profile of Paschen alpha in 3C 273. The spectrum is given as flux density in the observer's frame vs. velocity, with the data averaged into bins $20 \mathrm{~km} \mathrm{~s}^{-1}$ wide. The instrumental resolution is $\sim$ $750 \mathrm{~km} \mathrm{~s}^{-1}$. width of the hydrogen line. The linewidth of $P \alpha$ in 3C 273 is observed to be $3400 \mathrm{~km} \mathrm{~s}^{-1}$ full width at half maximum (FWHM), which is in good agreement with the linewidths of $3000-4000 \mathrm{~km} \mathrm{~s}^{-1}$ (FWHM) found for other hydrogen lines in this quasar (Baldwin 1975; Boksenberg et al. 1975; Boggess et al. 1979). The integrated rest frame intensity of the $P \alpha$ line is observed to be 7.2 $\times 10^{-13} \mathrm{erg} \mathrm{cm}^{-2} \mathrm{~s}^{-1}$, after subtraction of a flat baseline fitted through the observations between -7500 and $-6700 \mathrm{~km} \mathrm{~s}^{-1}$. This is in excellent agreement with the value of the rest frame $P \alpha$ line intensity in $3 \mathrm{C} 273$ found by Puetter et al. (1978), $7.8 \times 10^{-13} \mathrm{erg} \mathrm{cm}^{-2} \mathrm{~s}^{-1}$, and with that found by Grasdalen (1976), $9.0 \times 10^{-13} \mathrm{erg}$ $\mathrm{cm}^{-2} \mathrm{~s}^{-1}$. The line profile is very symmetric, with an upper limit of $10 \%$ on the difference between the integrated flux of the blue and red halves of the line profile. The measured center of the line is the same, within the uncertainties, as that predicted from a redshift of $z$ $=0.158$.

Figure 2 compares the $P \alpha$ line profile observed in 3C 273 with the profiles of $\mathrm{H} \beta, L \alpha$, and $\mathrm{C}$ IV in this quasar. In this comparison the $P \alpha$ profile has been smoothed over $380 \mathrm{~km} \mathrm{~s}^{-1}$, or half a resolution element. Boksenberg et al. (1975) find that their line profile of $\mathrm{H} \beta$ is contaminated on the red side of the profile by $\mathrm{Fe}$ II emission at velocities of +3900 and $+9700 \mathrm{~km} \mathrm{~s}^{-1}$, and by [O III] emission at a velocity of $+9000 \mathrm{~km} \mathrm{~s}^{-1}$. Similarly the profile of $L \alpha$, taken from Boggess et al. (1979), is contaminated by $\mathrm{N} \mathrm{v}$ emission on the red side of the profile, at a velocity of $+6400 \mathrm{~km} \mathrm{~s}^{-1}$. The profile of $\mathrm{C} \mathrm{IV}$, also taken from Boggess et al. (1979), is not affected by any other significant lines.

Figure 2 demonstrates that within the uncertainties of the measurements the line profile of $P \alpha$ is the same as those of $\mathrm{H} \beta, L \alpha$, and $\mathrm{C}$ iv. The comparison of $P \alpha$ and $\mathrm{C}$ iv shows that there is no difference between the red sides and blue sides of the line profiles for these two lines. The blue sides of the $\mathrm{H} \beta$ and $L \alpha$ profiles agree well with that of $P \alpha$, and within the uncertainties introduced by the emission from other lines, the red sides of the $\mathrm{H} \beta$ and $L \alpha$ profiles also agree well with that of $P \alpha$.

\section{Discussion}

Current models of quasars (Kwan and Krolik 1979, 1981; Canfield and Puetter 1980) attribute the observed hydrogen-line ratios to radiative transfer and optical depth effects within the dense broad-line-emitting clouds. In these models a relatively thin layer, on the cloud surface which is illuminated by the central ionizing source, is fully ionized by ultraviolet radiation, while the remaining cloud beyond the Strömgren radius is partially ionized by X-rays. Detailed calculations using this model are reasonably successful in explaining the observed $L \alpha / \mathrm{H} \beta$ ratios and other line ratios (Kwan and 

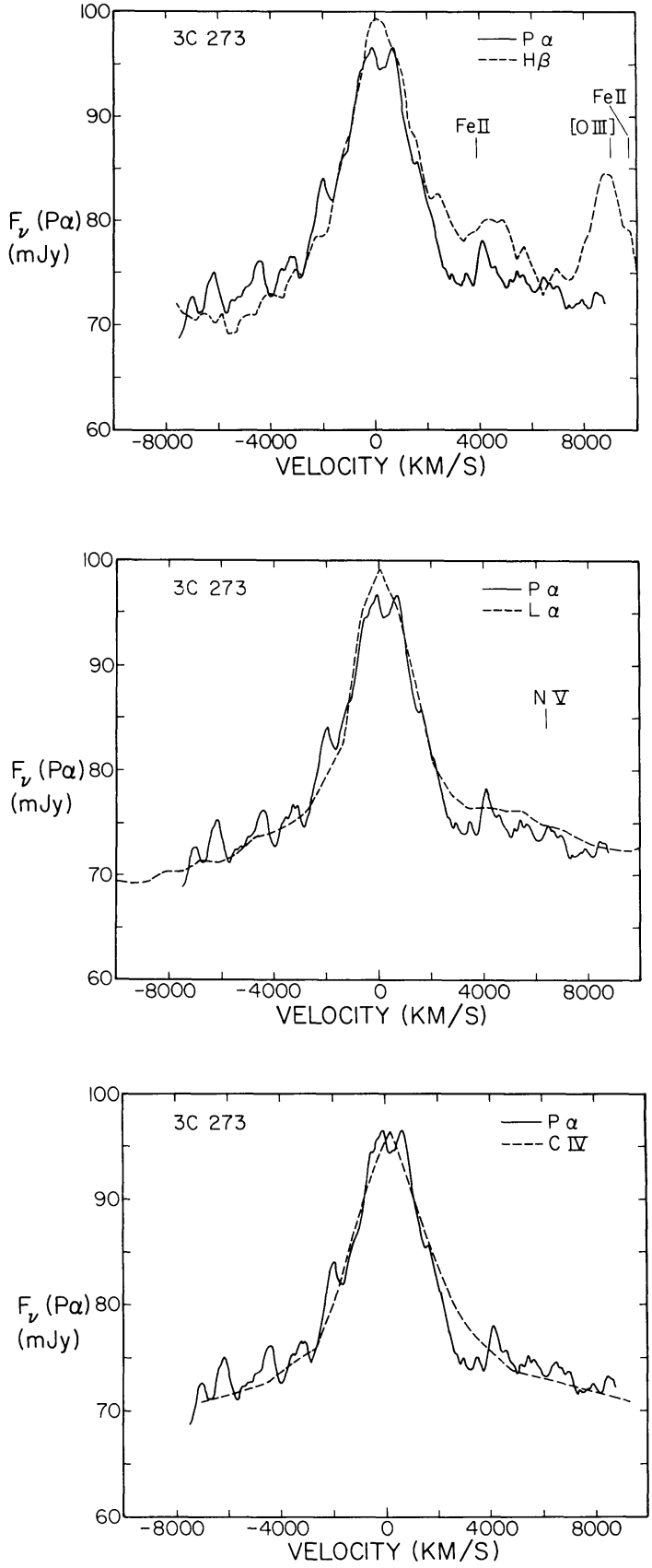

FIG. 2-The line profile (solid line) of $P \alpha$ in 3C 273, compared with the line profiles (dashed lines) of (a) $\mathrm{H} \beta$, (b) $L \alpha$, and (c) C IV $\lambda 1550 \AA$ in 3C 273. The spectrum of $P \alpha$ has been smoothed over $380 \mathrm{~km} \mathrm{~s}^{-1}$, or one-half the instrumental resolution. The instrumental resolution for the observations of $\mathrm{H} \beta$ (Boksenberg et al. 1975), $L \alpha$ (Boggess et al. 1979), and C IV (Boggess et al. 1979) were respectively 200, 1300, and $1000 \mathrm{~km}$ $\mathrm{s}^{-1}$. The scaling factor for plotting lines other than $P \alpha$ was determined by a weighted least-squares fit between $P \alpha$ and the other line, after subtraction of flat baselines fit through the data between -7500 and $-6700 \mathrm{~km} \mathrm{~s}^{-1}$. The line profile of $\mathrm{H} \beta$ (Fig. 2(a)) is contaminated by lines of $\mathrm{Fe}$ II and [O III], whose positions are marked. The position of $\mathrm{Nv}$, which contaminates the line profiles of $L \alpha$ (Fig. 2(b)), is also marked. Because of these contaminating lines, for $\mathrm{H} \beta$ and $L \alpha$ only the blue halves of the line profiles are used in the fits determining the scaling factors.
Krolik 1979, 1981; Canfield and Puetter 1980). The models predict very large $L \alpha$ optical depths within each cloud, and substantial optical depths in lines such as $\mathrm{H} \alpha$ and $\mathrm{H} \beta$. They also predict that most of the $L \alpha$ radiation that emerges from each cloud comes from the fully ionized zone, so that the cloud emits $L \alpha$ radiation essentially only from its illuminated face, while the other lines are produced predominantly in the extended partially ionized zone, and are radiated more or less isotropically by the entire cloud. If these models are correct, then the marked anisotropy of $L \alpha$ radiation from each cloud, compared with the relative isotropy of the radiation of the other lines, should make it possible to use line profile observations to constrain the possible velocity distribution of the numerous clouds within the broad-line region.

If the velocity distribution of the clouds in the broadline region is dominated by radial inflow or outflow, the model calculations (Kwan and Krolik 1981) would predict a large asymmetry between the relative strengths of the red and blue portions of the line profiles of $L \alpha$ and other lines, since the ratio of emitted intensities of $L \alpha$ and other lines differ by a factor of 100 between the front and back surfaces of the cloud. The good agreement of the line profiles of $L \alpha$ and $P \alpha$, as well as the agreement of the $L \alpha$ line profile with other hydrogenline profiles, indicates that either the velocity distribution is not primarily due to inflow or outflow, or that the clouds radiate $L \alpha$ more isotropically than is predicted by current models. The models also predict that $P \alpha$ and $\mathrm{H} \beta$ should originate mainly from the extended, partially ionized zone, but that $\mathrm{H} \beta$ should be more optically thick than $P \alpha$ (Kwan and Krolik 1981). The good agreement between the line profiles of $P \alpha$ and $\mathrm{H} \beta$ again argues that either the cloud motions are not primarily radial, or that differences in the optical depths of lines produced in the extended ionized zone do not substantially alter the angular distribution of emergent radiation from the cloud. The observations presented here of $P \alpha$ in 3C 273, as well as other observations of different line profiles in quasars (Davidson and Netzer 1979), thus indicate that if current models of radiative transfer within clouds are correct, the dominant velocity field of the clouds must be either rotational or random in nature. Alternatively, if the models of cloud kinematics of Weymann et al. (1982) and Blumenthal and Mathews (1979), arguing for radial outflow, are correct, then the predictions of anisotropic cloud emission in optically thick lines must be wrong. In any event it appears that a complete theoretical picture of the radiative transfer and kinematics of the broadline-emitting region is not yet consistent with the observations of the broad lines in quasars.

A final result of these observations is that the good agreement between the line profile of $P \alpha$ in $3 \mathrm{C} 273$, at a rest wavelength of $1.875 \mu \mathrm{m}$, and the profiles of other 
lines at substantially shorter wavelengths, indicates that possible reddening due to dust within the broad-line region has no differential effect on the visual and ultraviolet line profiles. The extinction at the rest wavelength of the $\mathrm{C}$ IV line, $1550 \AA$, should be 16 times that at $P \alpha$ for a normal extinction curve, so that if there existed significantly different amounts of reddening for radiation emitted at different velocities, there would be marked differences between the $\mathrm{C}$ IV and $P \alpha$ line profiles. Such differences are not observed. The ratio of the integrated fluxes of the blue and red halves of the line profile is the same, within 20\%, for $P \alpha$ and C rv. Similarly, the ratio of the line intensities of $P \alpha$ and $C$ IV at different velocities within the profile, after subtraction of the continuum, is essentially constant. Even in the wings, where the signalto-noise ratio of the line intensity compared to the continuum is lower, a conservative limit of a factor of 2.5 can be placed on variations in the ratio of $P \alpha$ to $\mathrm{C}$ IV with velocity. If the profiles of $P \alpha$ and $C$ IV are intrinsically identical, these limits lead to an upper limit of $E(B-V)<0$ m 02 on reddening differences between the blue and the red halves of the line profiles, and a limit of $E(B-V)<0$ m.12 on reddening differences between individual resolution elements within the line profile. The existence of dust which affects all velocities similarly cannot be ruled out from the line-profile observations presented here, although arguments have been advanced by Ennis et al. (1981) against the presence of significant amounts of dust in 3C 273.

\section{Conclusions}

Observations have been made of the line profile of $P \alpha$ in the quasar 3C 273. The $P \alpha$ line profile is the same, within the uncertainties, as the line profiles of $\mathrm{H} \beta, L \alpha$, and $\mathrm{C}$ IV. From this we conclude that if current models of broad-line-emitting clouds in quasars are correct, then the similarity of the $P \alpha$ line profile to the $L \alpha$ profile argues against a velocity field dominated by radial inflow or outflow. Alternatively, if the cloud motions are predominantly due to radial inflow or outflow, then the clouds must radiate more isotropically in $L \alpha$ than current models predict. The observed similarity of the line profiles of $P \alpha$, at $1.875 \mu \mathrm{m}$, and $\mathrm{C}$ IV, at $1550 \AA$, leads us to conclude that there is no differential reddening due to dust, between clouds moving at different velocities within the broad line region of $3 \mathrm{C} 273$.

We would like to thank our night assistant J. Carrasco at Palomar Observatory for help with the observations; D. Nadeau, J. L. Lacy, and T. R. Geballe for invaluable help with the equipment; and R. L. White for useful discussions. Financial support was provided by grants from NASA and NSF.

\section{REFERENCES}

Baldwin, J. A. 1975, Ap. J. 201, 26.

Blumenthal, G. R., and Mathews, W. G. 1979, Ap. J. 233, 479.

Boggess, A. et al. 1979, Ap. J. (Letters) 230, L131.

Boksenberg, A., Shortridge, K., Fosbury, R. A. E., Penston, M. V., and Savage, A. 1975, M.N.R.A.S. 172, 289.

Canfield, R. C., and Puetter, R. C. 1980, Ap. J. (Letters) 236, L7.

Davidson, A. F., and Netzer, H. 1979, Rev. Mod. Phys. 51, 715.

Elias, J. H. 1978, Ap. J. 223, 859.

Elias, J. H., Frogel, J. A., Matthews, K. M., and Neugebauer, G. 1982, A.J. 87, 1029.

Ennis, D. J., Soifer, B. T., Neugebauer, G., and Werner, M. W. 1981, Ap. Letters 22, 143.

Grasdalen, G. L. 1976, Ap. J. (Letters) 208, L11.

Kwan, J., and Krolik, J. H. 1979, Ap. J. (Letters) 233, L91. 1981, Ap. J. 250, 478.

Nadeau, D. 1980, Ph.D. Thesis, California Institute of Technology.

Puetter, R. C., Smith, H. E., Soifer, B. T., Willner, S. P., and Pipher, J. L. 1978, Ap. J. (Letters) 226, L53.

Weymann, R. J., Scott, J. S., Schiano, A. V. R., and Christiansen, W. A. 1982, Ap. J. 262, 497. 